\title{
A Global consortium, next-generation SARS-CoV-2 antibody therapeutics and stabilized spike \\ Erica Saphire ${ }^{1}$ \\ ${ }^{1}$ No affiliation given \\ erica@lji.org
}

\begin{abstract}
Although vaccines against SARS-CoV-2 are highly effective, five months after their release a majority of the world's population remains unvaccinated. Monoclonal antibodies have shown efficacy for individuals infected with SARS-CoV-2, but those in clinical trials were isolated early in the outbreak and can be less effective against emerging Variants of Concern (VoCs), demonstrating that first-in-class is not always best-in-class. The Coronavirus Immunotherapeutic Consortium (CoVIC) is an international effort to conduct side-by-side comparisons of over 300 leading therapeutic antibody candidates against the SARS-CoV-2 spike protein contributed by large and small companies and academic labs. Competition binning of the mAbs revealed a landscape of antibody binding footprints. This, together with pseudovirus neutralization assays highlight antibody pairings for potent therapeutic antibody cocktails that are resistant to VoCs and have low cost of goods. Our group also undertook an antibody discovery effort using the Berkeley Lights Beacon instrument with structural biology-grade antigens to isolate highly potent antibodies from COVID-19 patients that are effective against VoCs. We also engineered a nextgeneration Spike protein, "VFLIP", which stably maintains the prefusion conformation needed to elicit potent neutralizing antibodies without the need for a potentially immunogenic exogenous trimerization domain. The resistance of VFLIP to a range of temperatures is valuable for use as an immunogen, and its ability to bind antibodies that "split" spike trimers make it an important tool for structural analyses.
\end{abstract}

